\title{
Characterization of a Low Profile, Rapidly Deployable, MEMS Pressure Sensor Array for Aerodynamic Applications
}

\author{
Senthilkumar Subramanian ${ }^{1}$ Rory Hampson ${ }^{2}$ and Dayi Zhang ${ }^{3}$ \\ ${ }^{1}$ University of Glasgow, Glasgow, G12 8QQ, UK \\ ${ }^{2,3}$ University of Strathclyde, Glasgow, G1 $1 X Q, U K$
}

\author{
Konstantinos Kontis ${ }^{4}$, Gordon Dobie ${ }^{5}$, Charles Macleod ${ }^{6}$ and Jae Son ${ }^{7}$ \\ ${ }^{4}$ University of Glasgow, Glasgow, G12 8QQ, UK \\ 5,6 University of Strathclyde, Glasgow, G1 1XQ, UK \\ ${ }^{7} P P S$ UK Limited, G1 1RD, UK
}

\begin{abstract}
A low profile, rapidly deployable (and redeployable), air pressure sensor for the use in aerodynamic measurement, aerodynamic system optimization and non-invasive validation of aerodynamic theory in motorsport and aerospace was developed and tested for subsonic aerodynamic applications. Three cases - Constant pressure, External flow and Internal flow experiments were considered to validate the performance and accuracy of the sensors. The experiment results demonstrated that this system is accurate, compatible, time saving and non-intrusive for most subsonic aerodynamic applications. The effect of sensor thickness on the flat plate boundary layer was investigated using PIV and the results are presented. New design iteration are proposed to optimize the design.
\end{abstract}

\section{Nomenclature}

$\begin{array}{ll}m & =\text { meter } \\ \mathrm{Hz} & =\text { Hertz } \\ \mathrm{Pa} & =\text { Pascal } \\ \mathrm{kPa} & =\text { Kilopascal } \\ \mathrm{L} / \mathrm{D} & =\text { Length to Diameter Ratio } \\ C_{P} & =\text { Coefficient of Pressure }\end{array}$

\section{Introduction}

Conventional static pressure measurement techniques require ports to be drilled into the body surface, making the component unsuitable for use after testing. Furthermore, the static ports are connected with series of tubes in order to transport the fluid pressure to a pressure measurement transducer. This method is cumbersome when applied to complex shaped models and high value parts such as Formula 1 car front wings [1], and modern fighter aircraft wings particularly when the parts are small has variable geometry [2]. Despite the cost and complexity, pressure measurement plays a significant role in fluid dynamics research, and so this research aims

\footnotetext{
${ }^{1} \mathrm{PhD}$. Candidate, James Watt School of Engineering, University of Glasgow, UK.

${ }^{2}$ Research Associate, Electronic \& Electrical Engineering, University of Strathclyde, UK.

${ }^{3}$ Research Associate, Electronic \& Electrical Engineering, University of Strathclyde, UK.

${ }^{4}$ Professor of Aerospace Engineering, James Watt School of Engineering, University of Glasgow, UK. AIAA

Fellow

${ }^{5}$ Reader, Electronic \& Electrical Engineering, University of Strathclyde, UK.

${ }^{6}$ Senior Lecturer, Electronic \& Electrical Engineering, University of Strathclyde, UK.

${ }^{7}$ Pressure Profile Systems, USA.
} 
to develop and characterise a rapidly deployable, and non-invasive, pressure measurement system to simplify and improve the cost-efficiency of aerodynamic system development. The method proposes an array of pressure sensors in a 'strip' that can be fixed temporarily onto any complex 3-dimensional bodies without the need of altering/damaging the testing body. This sensor system is in early stages of development, and so laboratory characterisation and evaluation of its suitability for use is performed in this paper, prior to further development and deployment onto application specific structures, particularly in aerospace and automotive design [3] where the simple and non-invasive nature of the sensor can have the highest impact.

\section{Sensor System Overview and Application}

The sensor used in this characterisation is a PPS Aerostrip $400 \mathrm{~mm} \mathrm{1x12} \mathrm{element} \mathrm{pressure} \mathrm{sensor} \mathrm{array}$ prototype [4]. This is a low profile, rapidly deployable (and redeployable), air pressure sensor array intended for use in direct aerodynamic measurement, aerodynamic system optimisiation, and non-invasive validation of aerodynamic theory/CFD in motorsports and aerospace. The sensor array features a low-profile adhesive cover, to minimise disturbance to airflow and to allow the array to be fixed securely to complex geometry surfaces without the need for drilling as would be required by pressure taps. The array can also be removed from the structure without damage, limiting the need for duplication of components. This has the potential to save both time and money in the development, characterisation, and validation of aerodynamic systems in academia and industry.

The sensor arrays, illustrated in Fig. 1, can be combined into larger arrays, or used independently in up to 4 separate areas of the structure under test by combining the strips together using a PPS U400 DAQ controller. In future models this is envisaged to be fully wireless, to eliminate the need for cables, however in its current form the cables are kept downstream and are low profile so as to not impede the measurements. The sensors can conform to complex geometry and multiaxial curvature that is inherent in many aerodynamic systems, with a bend radius limited to $>5 \mathrm{~mm}$, making the system suitable for a wide variety of applications in the laboratory and real world. The suitability of which shall be investigated in this paper.

The sensor data is streamed at a maximum rate of $200 \mathrm{~Hz}$, through the DAQ controller, to a PC or laptop running LabView for further integration into customised systems and measurement apparatus. Here the data can be visualised graphically, saved, and processed further to suit the users/systems requirements.

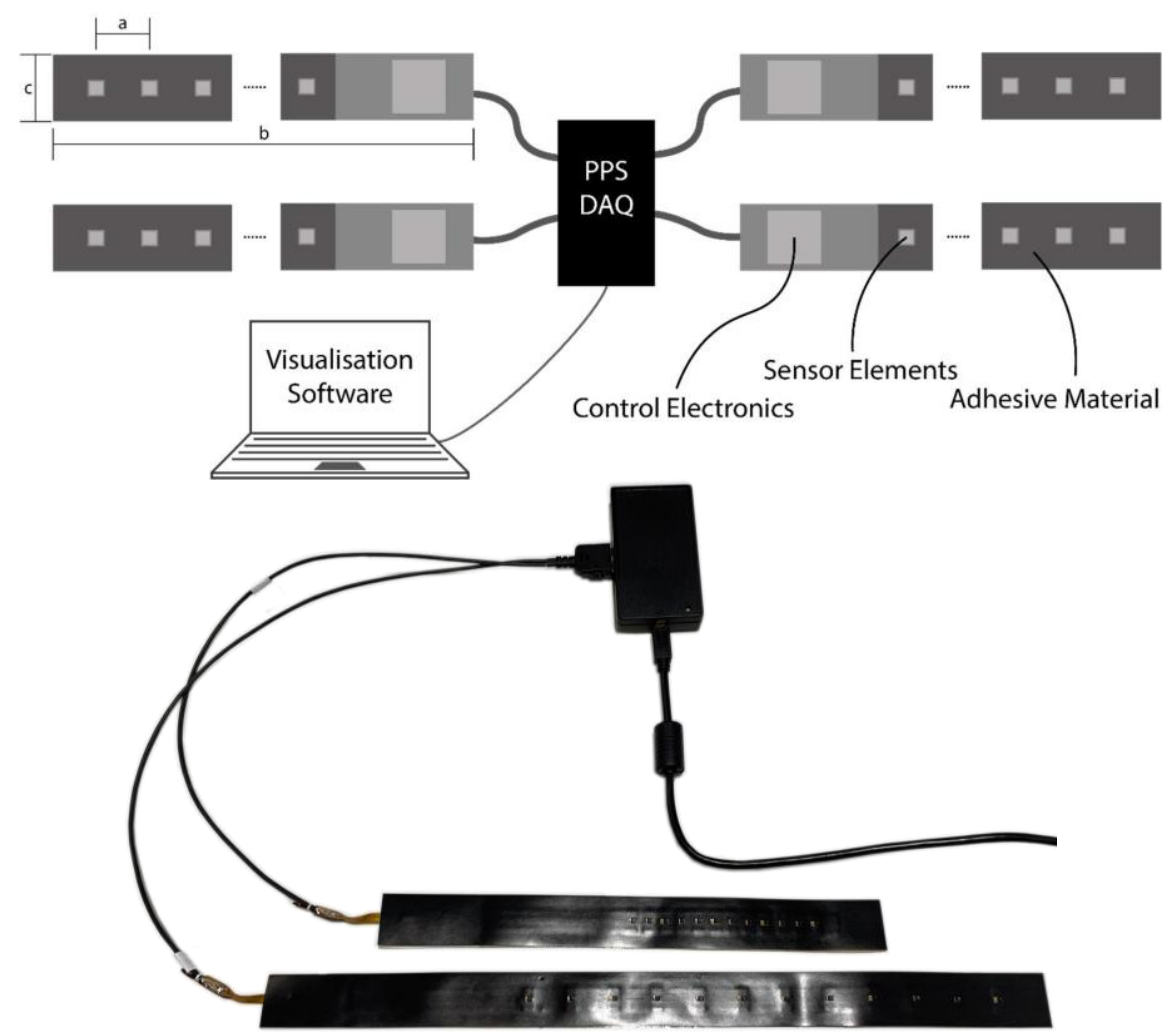


Fig. 1 Working schematic of an integrated PPS Aerostrip system. Up to 4 wired sensors can be combined, either independently or as part of a larger array, communicating with a GUI and recorder on a PC via a custom DAQ. Dimensions are defined in the table of specifications.

Table 1 - Prototype sensor general specifications

\begin{tabular}{|l|l|l|}
\hline \multicolumn{2}{|c|}{ AEROSTRIP PROTOTYPE SENSOR SPECIFICATIONS } \\
\hline VARIABLE & \multicolumn{1}{|c|}{ VALUE } & \multicolumn{1}{c|}{ UNITS } \\
\hline Rating Range & $26-126$ & $\mathrm{kPa}$ (Absolute) \\
\hline Resolution & $1 / 40960$ & $\mathrm{kPa}$ \\
\hline Sample Rate & 200 & $\mathrm{~Hz}$ \\
\hline No. Elements & 12 & - \\
\hline Sensor Pitch (a) & 25 & $\mathrm{~mm}$ \\
\hline Sensor Strip Length (b) & 400 & $\mathrm{~mm}$ \\
\hline Sensor Width (c) & 10 & $\mathrm{~mm}$ \\
\hline Sensor Thickness & 1.4 & $\mathrm{~mm}$ \\
\hline Calibrated Temperature Range & $-40<\mathrm{T}<85$ & ${ }^{0} \mathrm{C}$ \\
\hline
\end{tabular}

\section{Sensor Specifications}

The sensors have the general technical specifications detailed in Table 1. The sensors are temperature compensated over the stated operating range and have a temperature coefficient $<0.7 \mathrm{~Pa} /{ }^{\circ} \mathrm{C}$ over the full range. This is important when dealing with real world applications where accuracy at low temperatures is expected. The sensor sample rate limits the use of the system to measurement of vortex shedding at high frequencies; however, vortex separation of $<100 \mathrm{~Hz}$ can be measured without issue. Other sensor variants are available with lower sensor pitches for higher spatial resolution to the measurements, at the cost of coverage area. The sensor accuracy is not affected by this. It is important to note that the accuracy of the sensor is quoted for static conditions, and hence the need

this work to characterise the performance in dynamic flow measurements in real applications.

\section{Validation method}

Four different incompressible flow conditions were considered to validate the performance and accuracy of the sensors in laboratory settings. These were:

1. Constant pressure inside a plenum chamber with constant inlet and exit flow rate,

2. External flow around a 2-dimensional cylinder,

3. Internal flow through a 3-dimensional S-duct,

4. PIV investigation on the Boundary Layer over PPS Strip.

A 48 channel ZOC 23B Scanivalve pressure scanner [5] was used to measure the wall static pressure of the cylinder and S-duct, which were then compared with the results of PPS sensors recorded in LabView. The calibration of the pressure scanner was carried out using a Druck DPI 610/615 Portable Pressure Calibrator [6]. All the experiments were conducted at the University of Glasgow Acre Road wind tunnel facility.

\section{Results and Discussion}

\section{A. Constant Pressure inside a plenum chamber with constant inlet and exit flow rate}

For the constant flow experiment, the inlet and outlet flow rates of a stainless-steel plenum chamber of $0.138 \mathrm{~m} 3$ volume were controlled to get a constant pressure inside the chamber. The static pressure of the chamber is monitored by a PXM319 sensor [7] attached to the side wall. The PPS sensor arrays were securely fixed at the flat lower wall of the chamber. The pressure measurement of the PPS sensors at constant pressure is presented in Fig. 2. The result show good agreement in all the sensing elements of the sensor strip, corresponding properly 
with expectation from the reference sensor. This provided confidence, from the sensor stability, to move onto more complex geometries.
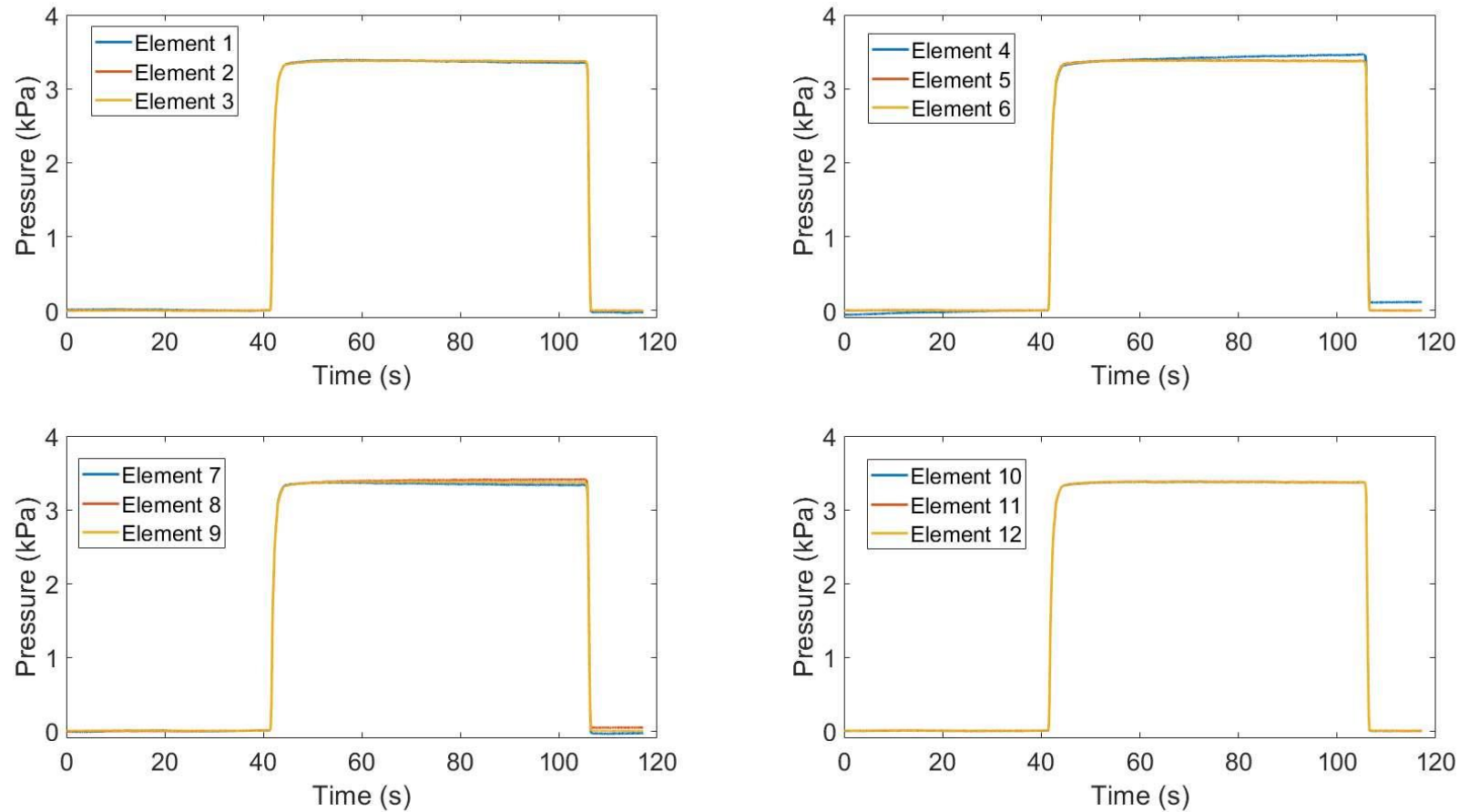

Fig. 2 Static pressure measurement of different sensing elements in a single sensor strip

\section{B. External flow around a 2-dimensional cylinder}

Flow over a circular cylinder is a simple yet useful model to compare the pressure at the attached, transition, and wake regions of the flow [8]. A 2-Dimensional cylinder of radius $0.08 \mathrm{~m}$ was fixed vertically inside a $2.1 \mathrm{~m} \times 1.5 \mathrm{~m}$ closed-circuit Handley-Page wind tunnel test section, operated at flow rates of $11.6 \mathrm{~m} / \mathrm{s}, 16.2 \mathrm{~m} / \mathrm{s}$ and $21.1 \mathrm{~m} / \mathrm{s}$ respectively for the tests. The PPS sensor strips were affixed in such a way that the $1 \mathrm{st}$ and 10 th sensor elements coincided with the front and rear stagnation points, and the 5th sensor element coincided with the 90degree point of the cylinder. Fig. 3, 4 and 5 show good agreement between the Scanivalve pressure transducer and the PPS sensor at the three specified flow rates respectively. Small variations in the PPS sensor outputs, with respect to the reference sensor, around the 90-degree point, at all test velocities, were attributed to the local increase in the width of the cylinder due to the thickness of sensor strip. The PPS sensors show good correlation with the reference sensors, providing additional confidence for further analysis and deployment of the sensors to more complex structures.




Fig. 3 Comparison of Cp (Coefficient of Pressure) distribution around Cylinder at $11.6 \mathrm{~m} / \mathrm{s}$

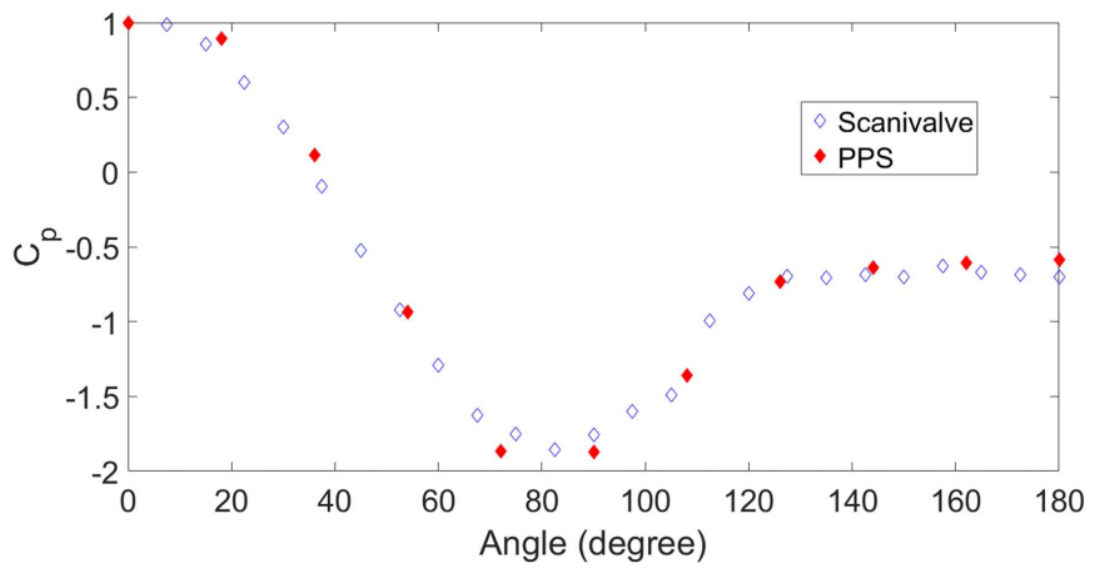

Fig. 4 Comparison of $\mathrm{Cp}$ (Coefficient of Pressure) distribution around Cylinder at $16.2 \mathrm{~m} / \mathrm{s}$

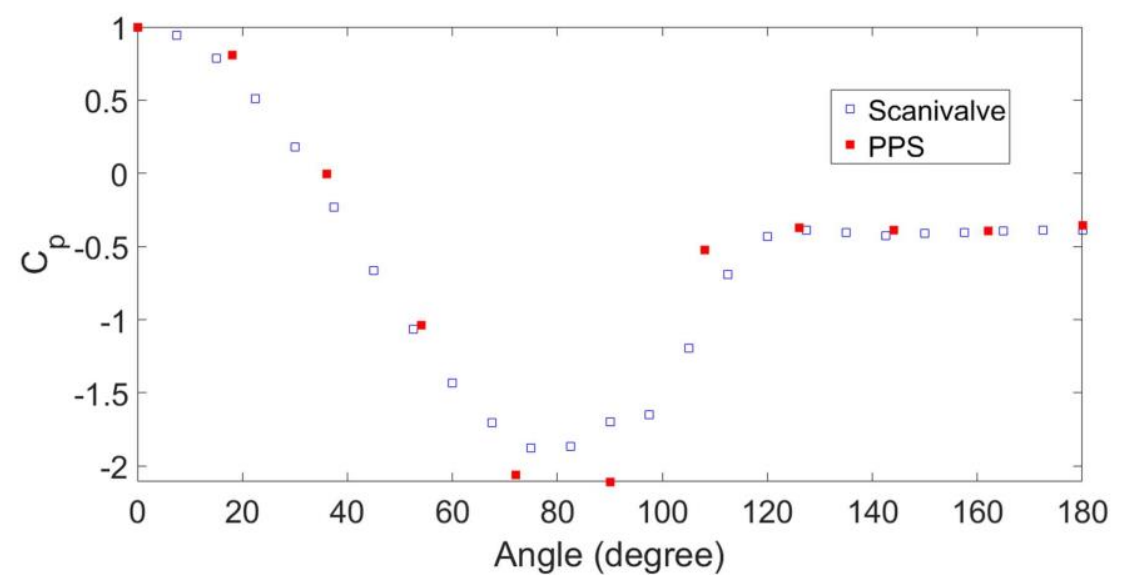

Fig. 5 Comparison of Cp (Coefficient of Pressure) distribution around Cylinder at $21.1 \mathrm{~m} / \mathrm{s}$

\section{Internal flow through a 3-dimensional S-duct.}

A fully instrumented S-shaped duct rig comprising of 43 static pressure holes to obtain the internal static pressure distribution was used in this experiment, to validate sensor performance on complex geometry, as shown diagrammatically in Fig. 6. The duct static pressure holes were located at equiangular spacings of $0,60,120$ and 180-degrees with 14 static pressure holes at 0 and 180-degrees, and 12 and 13 static pressure holes on 60 and 120degrees respectively as shown in Fig. 6, and Fig. 7. The static pressure holes were connected to a 48 channel ZOC $23 \mathrm{~b}$ Scanivalve pressure scanner and the time averaged static pressure measurements for all the channels were recorded. The L/D ratio of the S-duct is 5 and the diffusion area ratio of A throat/A exit is 0.8 . The duct was connected to two straight sections, one at the inlet and other at the outlet, that help with the development of the boundary layer at the inlet and to align the flow to the engine axis at the exit $[9,10]$. The exit of the duct was connected to a $50 \mathrm{JM}$ Maxfan 2-stage axial fan with dynamic speed control. The comparison experiment was performed at 18, 22 and 27 $\mathrm{m} / \mathrm{s}$ respectively. 


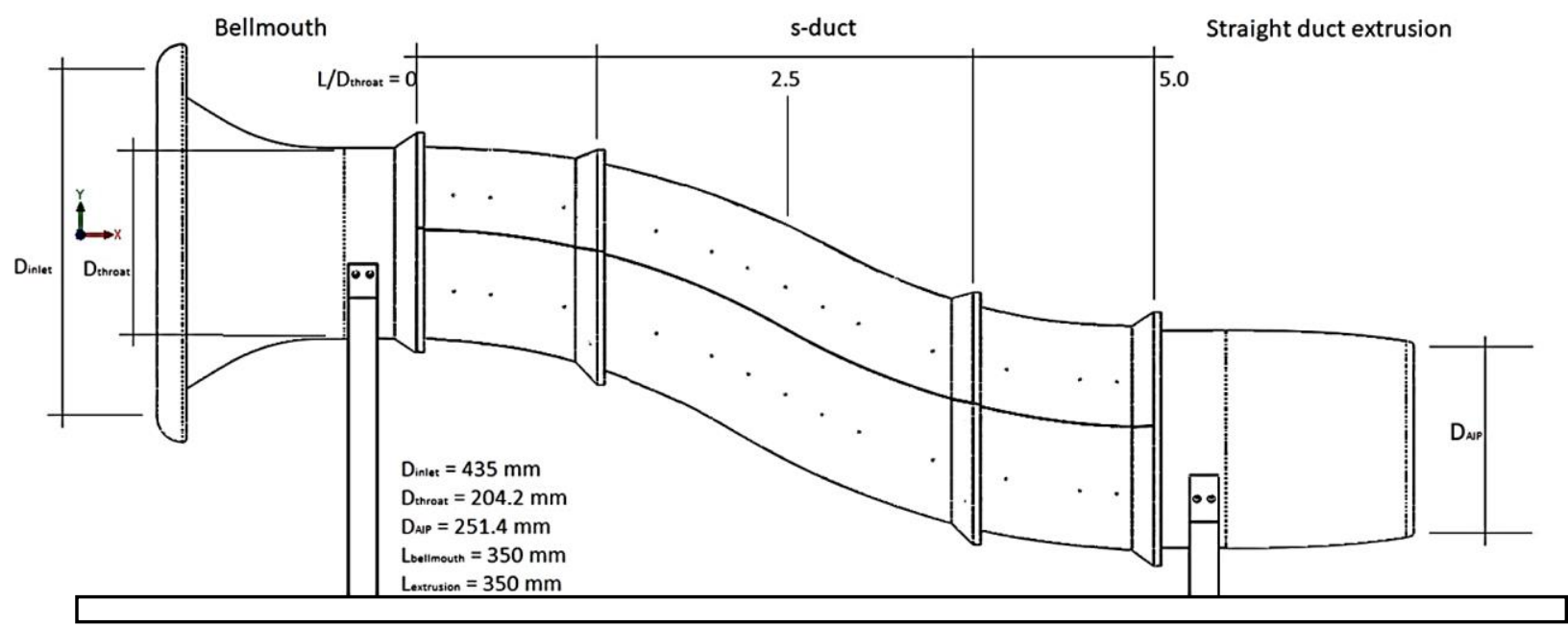

Fig. 6 S-duct assembly with static pressure holes

4 identical PPS sensor strips, each having 12 sensing elements were fixed streamwise along the inside walls of S-duct at 4 angular locations shown in Fig. 7, and the PPS Aerostrips are fixed in such a way that the strip sensors are coincide with the static pressure holes as shown in Fig. 8. A single strip can cover about 1/3rd of the total duct length. Hence to get complete pressure distribution along the duct length, the experiments were repeated three times shifting the strips at locations streamwise on each run. The change in the ambient pressure conditions on each run is corrected relative to the sea level conditions.

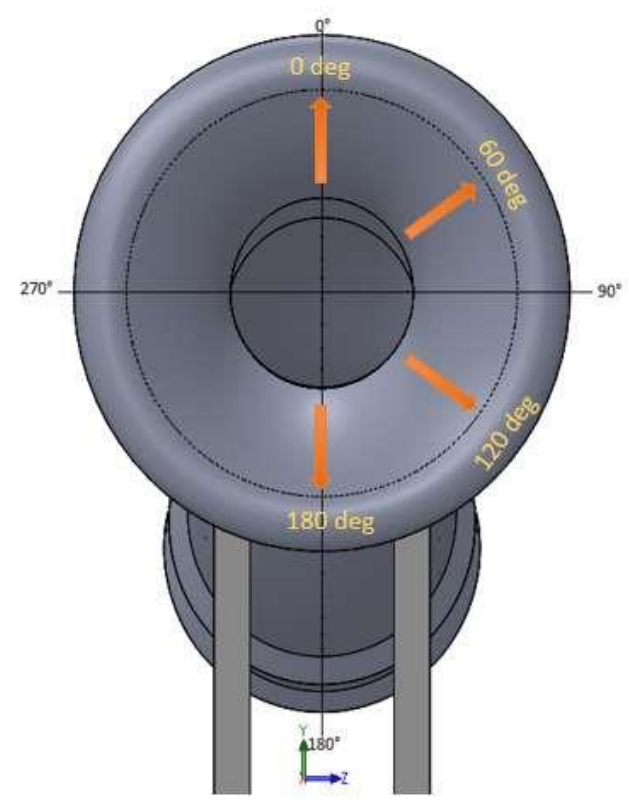

Fig. 7 Frontal view showing the streamwise location of static pressure holes. 


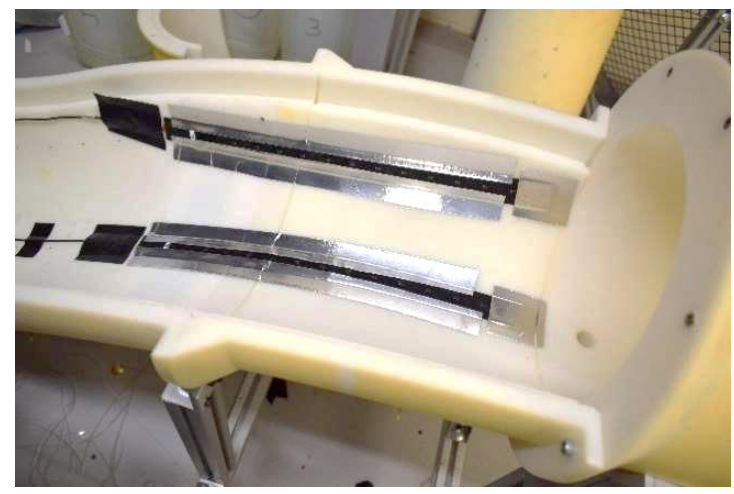

(a)

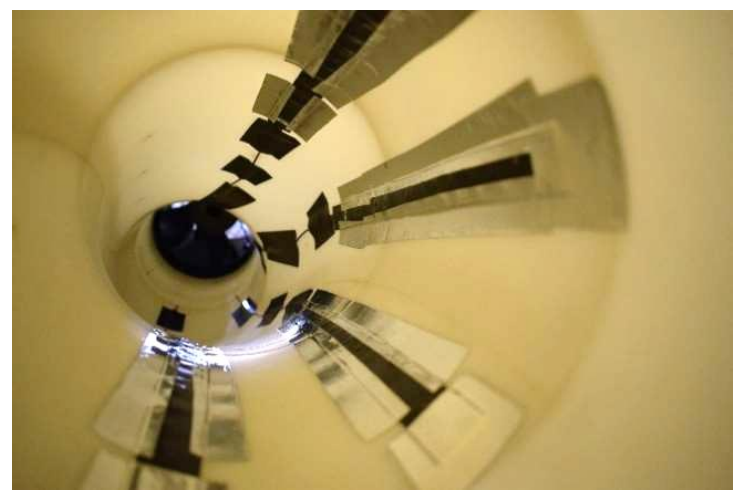

(b)

Fig. 8 PPS Aerostrip sensors fixed inside S-duct.

The top wall static pressure distribution along the length of S-duct for the three different velocities were compared with the measured PPS sensor values as shown in Fig. 9. The PPS sensor corresponds well with low pressure at the entrance of the duct, and the high-pressure region through the bends of the diffuser [11], at all flow velocities tested. This is a positive result, showing that the PPS sensor is capable of measuring pressure within components with complex 3D geometries in a meaningful manner. Similar to the $2 \mathrm{D}$ cylinder experiment result, small variation in the sensor output has been observed around convex surface of S-Duct.

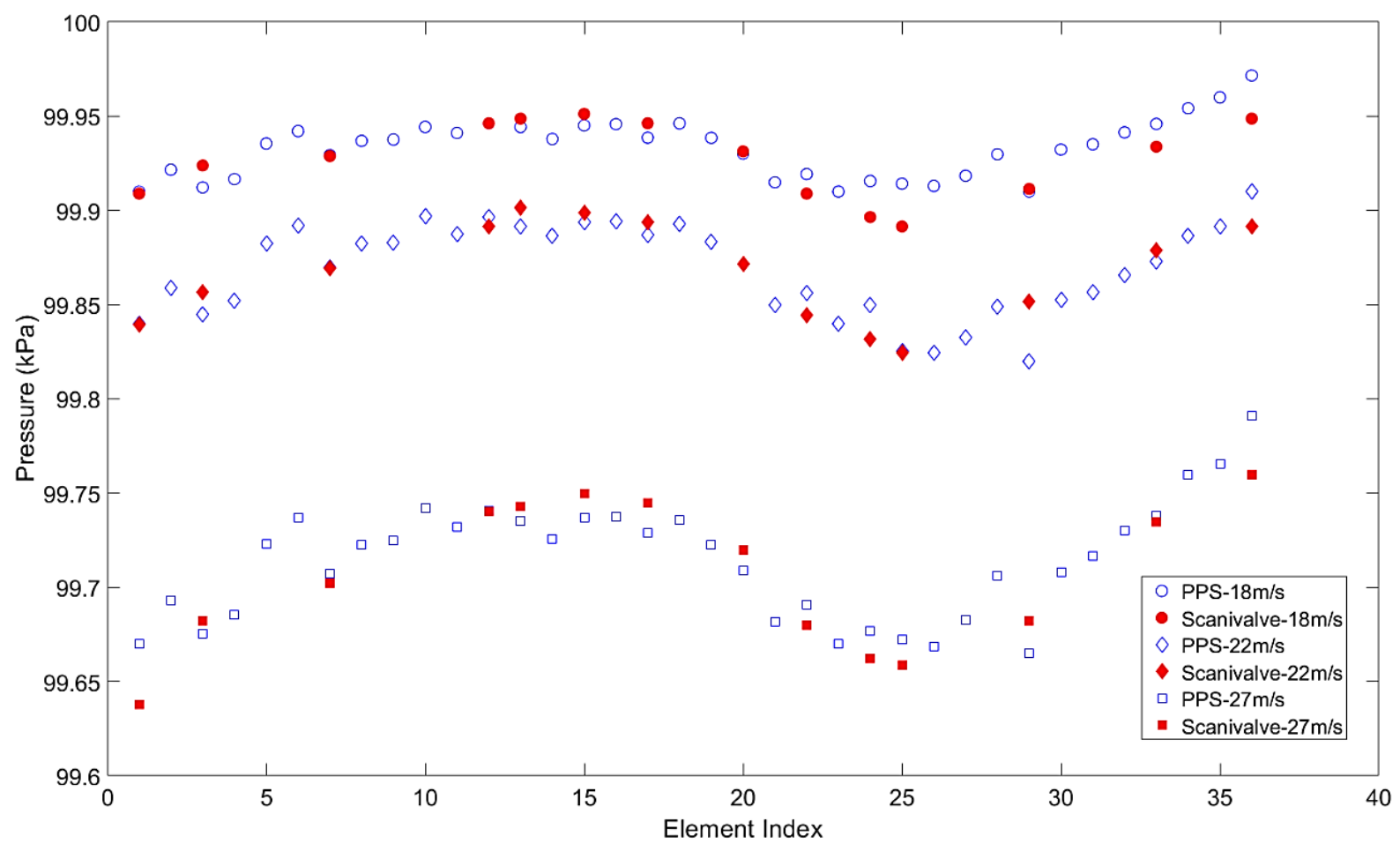

Fig. 9 Comparison of streamwise static pressure variation along 0-degree axis (from inlet to exit)

The static pressure distribution at 60-degree angles, shown in Fig. 10, slightly overestimates the pressure in the low-pressure regime, possibly due to misalignment of the straight strips from static pressure holes as a result of the 3-dimensional curved profile. The correlation at the high-pressure end of the duct is considerably better, where the alignment of the strips coincides with the pressure holes. The results are still generally positive, with the change in flow velocity being appropriately tracked, however the strip positioning accuracy deep in the s-duct limits the agreement, as expected in this type of experiment. Further testing can address this issue. A similar 
situation encountered at 120-degree angles, where the slight misalignment in the accuracy of straight strip positioning with the curved profile of S-Duct resulted in overestimation of pressure distribution at lower end as shown in Fig 11.

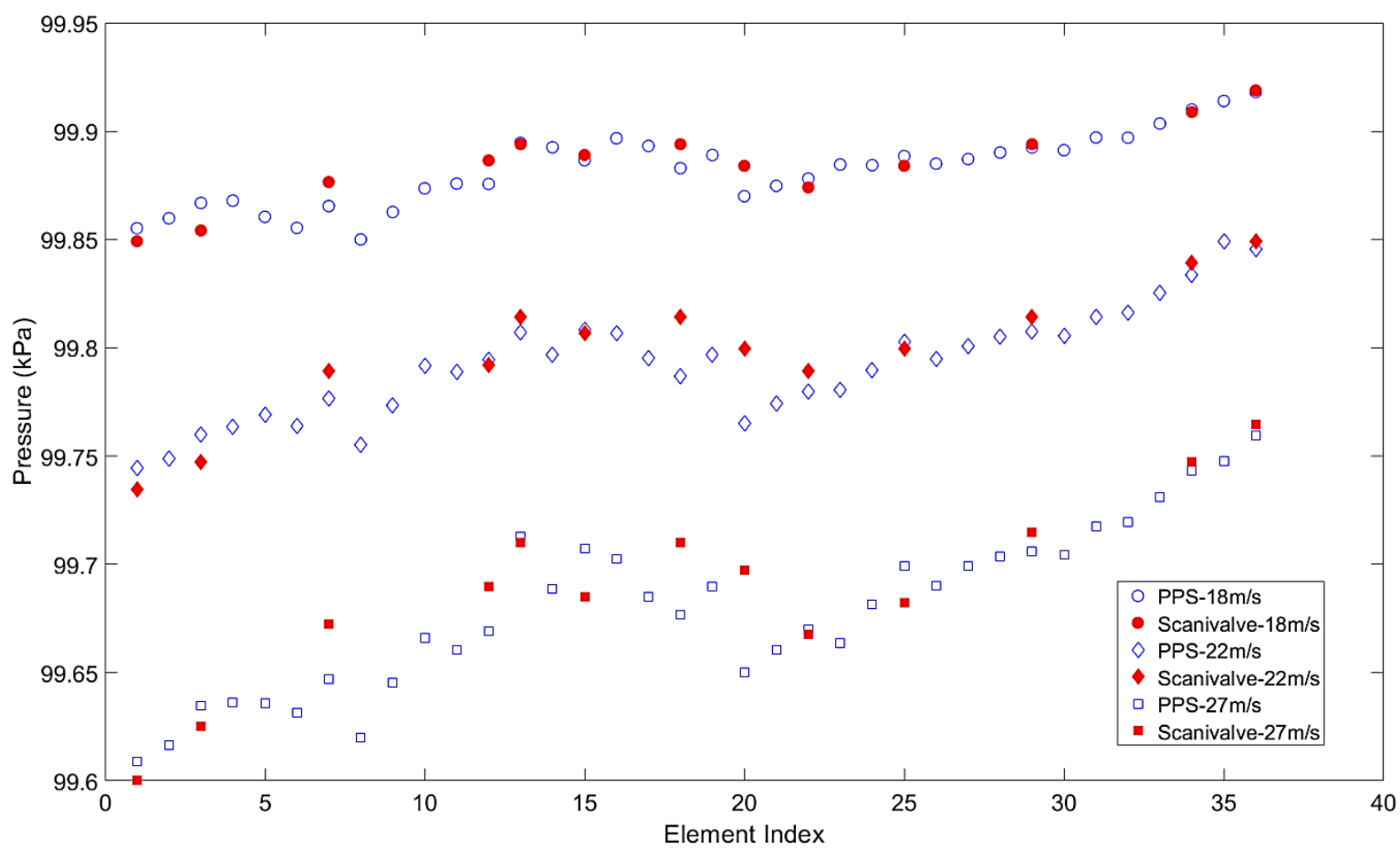

Fig. 10 Streamwise static pressure distribution along 60-degree axis (from inlet to exit)

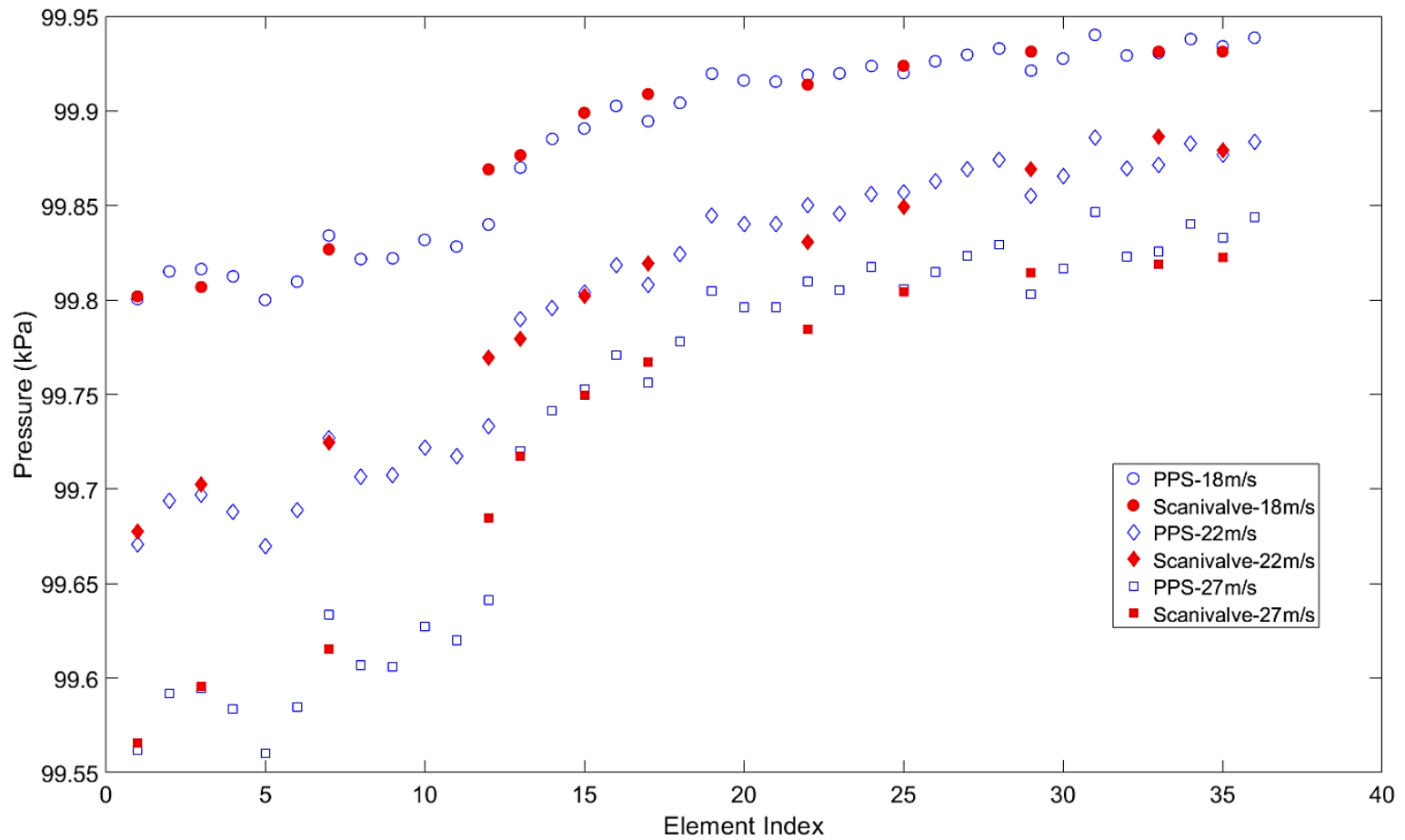

Fig. 11 Streamwise static pressure distribution along 120-degree axis (from inlet to exit) 
Contrary to the side angles $-60 \mathrm{deg}$ and $120 \mathrm{deg}$, the streamwise pressure distribution measured by PPS Aerostrip along the S-Duct at 180 degree is fairly consistent with the Scanivalve pressure scanner measurements at all the three velocities.

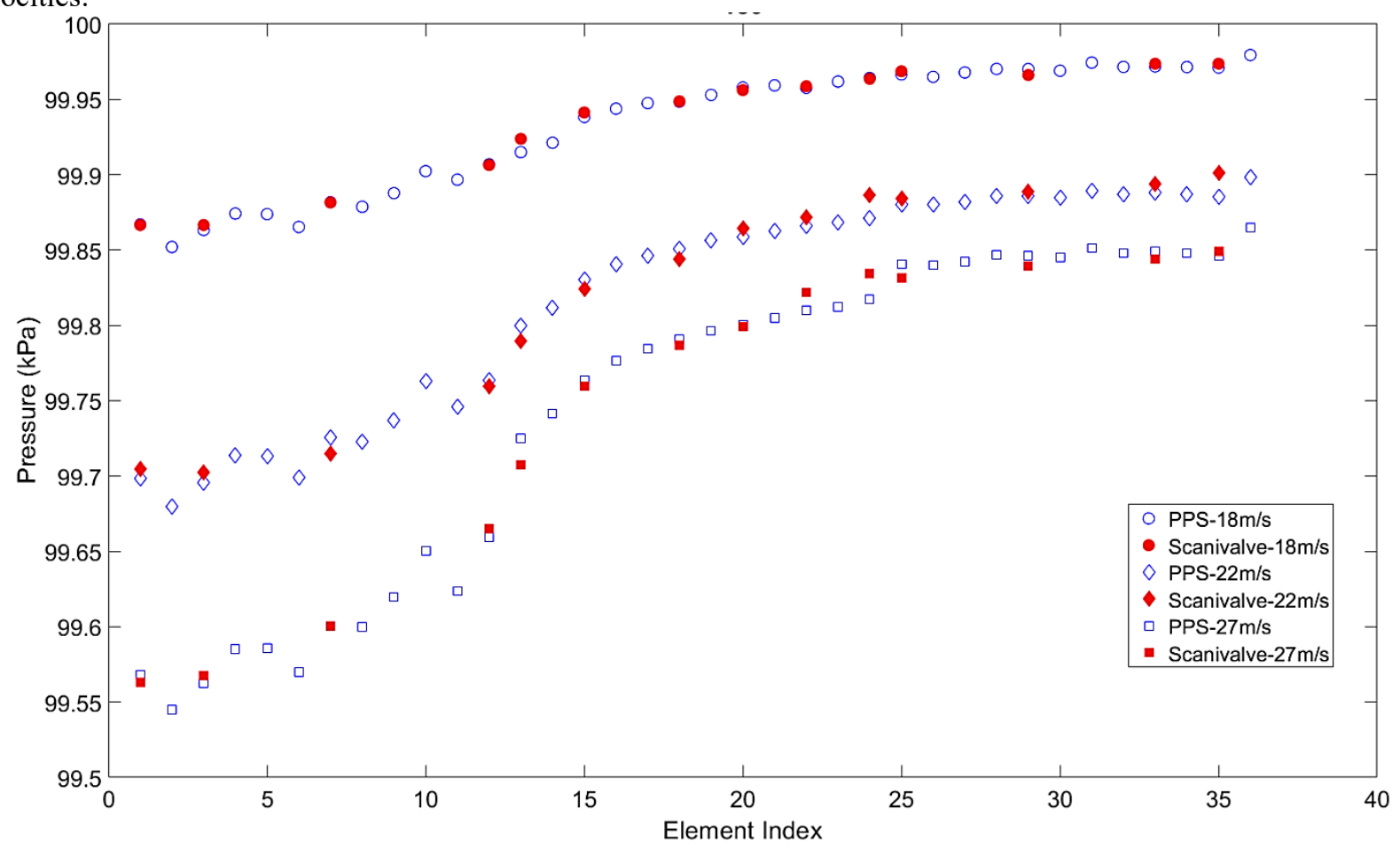

Fig. 12 Streamwise static pressure distribution along 180-degree axis (from inlet to exit)

From the results of both the 2D cylinder and 3D S-Duct experiments, it is observed that the accuracy of PPS Aerostrip is flawlessly matched with the Scanivalve pressure measurements at stagnation points, wake region and other curved surfaces. However, the accuracy is slightly varying at points where the flow needs to pass horizontally over the top of convex surfaces. Although, the thickness of the sensor is kept as low $(1.4 \mathrm{~mm})$, it is suspected that the small increment in thickness at the tip of concave surface would moderately increase the local velocity and in turn reduce the local pressure in that region. To investigate this, a Particle Image Velocimetry (PIV) experiment was planned to detect the change in local velocity due to addition of sensor thickness.

\section{PIV investigation on the Boundary Layer over PPS strip.}

Particle image velocimetry (PIV) is performed on the PPS Aerostrip to understand the effect of strip thickness on the flat plate surface boundary layer using a purpose-built setup. This setup consists of a plenum chamber that is pressurised to $4 \%$ above atmospheric pressure. A $16.76 \mathrm{~mm}$ exit diameter nozzle is mounted to one of the chamber walls such that the nozzle inlet sits inside the chamber while the outlet will expel the air into the room. This nozzle has been designed to expel a uniform flow and has been manufactured using rapid prototyping using a Formlabs printer. The PPS strip fixed on a flat plate model sit at the near field flow development jet boundary region. The chamber is filled with oleic particles using a seeder. A laser sheet is generated using a Litron LDY301 YLF laser at a frequency of $0.5 \mathrm{kHz}$. The laser sheet is parallel with the nozzle exit plane and illuminates the particles. A Photron APX-RS high-speed camera is used to capture the moving particles at $1000 \mathrm{fps}$. The laser and camera are synchronized using LaserPulse Synchronizer model 610035. A turbulent flow of Jet velocity $84 \mathrm{~m} / \mathrm{s}$, corresponding to Reynolds number $9.51 * 10^{4}$ was achieved at the exit of the nozzle. This setup is shown in Fig. 13 and 14. 


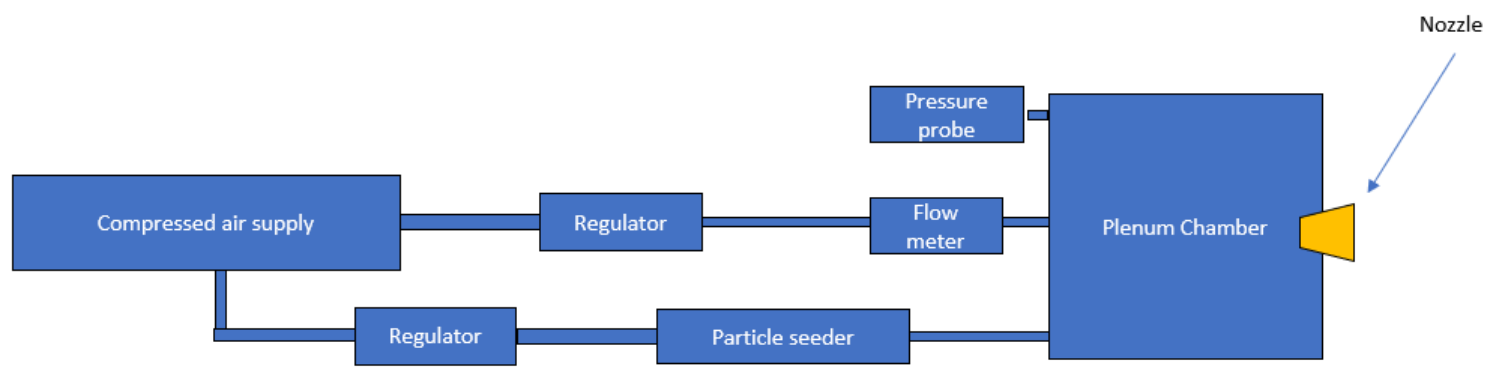

Fig 13. Schematic of the setup upstream of the nozzle

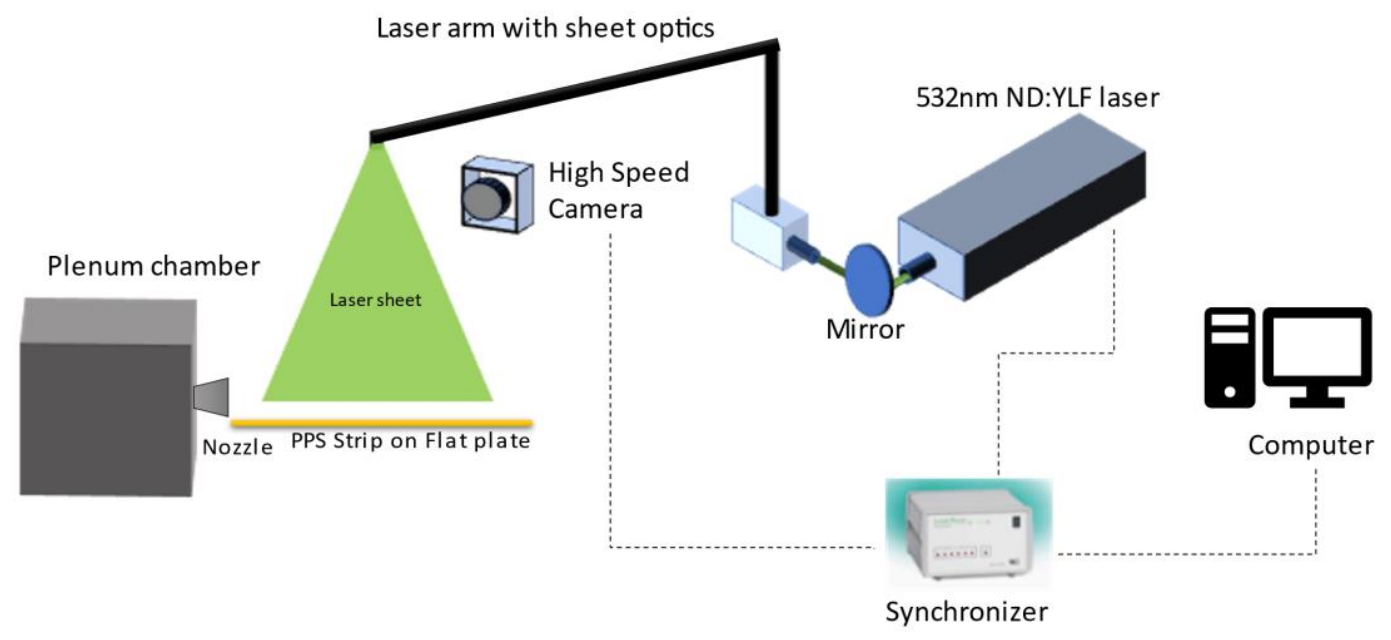

Fig 14. Schematic of the setup downstream of the nozzle

An initial experiment was performed using a wide angle $(50 \mathrm{~mm})$ lens on flow over flat plate with and without PPS Aerostrip. The result of the experiment shows the addition of the sensor strip had a little effect on the boundary layer as seen on Fig. 15 and Fig. 16. The presence of successive sensors along the Aerostrip can be seen as small ridges on the flat plate as in the PIV image in Fig. 16. The difficulty in observing the fine details of the change in flow features due to the presence of sensors can be overcome by replacing the wide lens with a telephoto lens. The addition of 
telephoto lens of $105 \mathrm{~mm}$ focal length zooms into the flow and provides a detailed velocity distribution over the sensors as shown in Fig, 17 and Fig. 18,

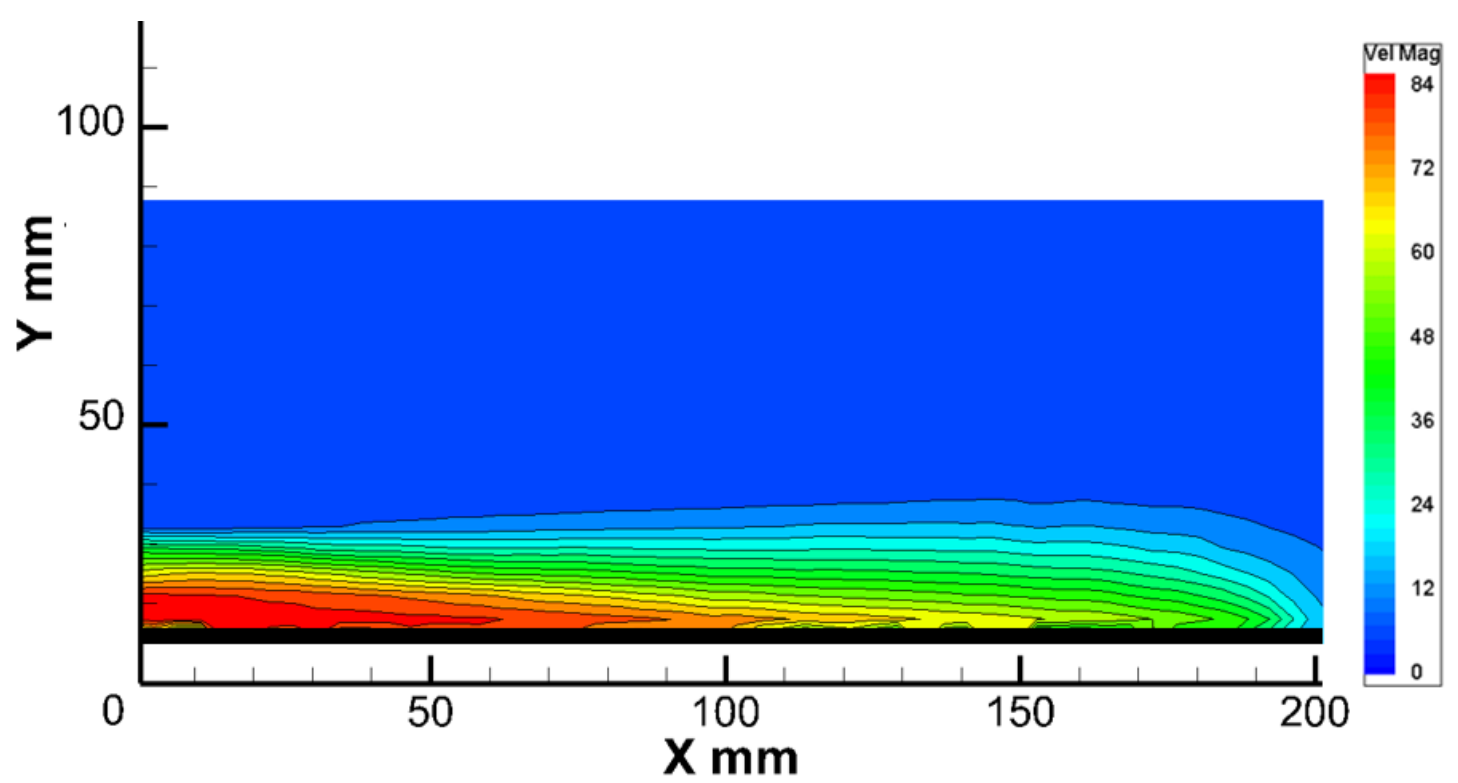

Fig. 15 Streamwise velocity distribution along $X$ axis over flat plate without PPS Aerostrip



Fig. 16 Streamwise velocity distribution along $X$ axis over flat plate with PPS Aerostrip

The Fig. 18 focuses flow over 3 successive sensors. The acceleration of flow over the sensor elements and streamwise velocity component disturbance can be seen in the Fig. 18. Unlike in cases where presence of bump produces adverse pressure gradient ensuring flow separation $[12,13]$, the flow over PPS Aerostrip produce stable 
regions ahead and behind sensors. Moreover, the rounded and fuller shape of the sensor hump decreases the possibility of flow separation in contrast to sharp edged hump in subsonic flow [13].

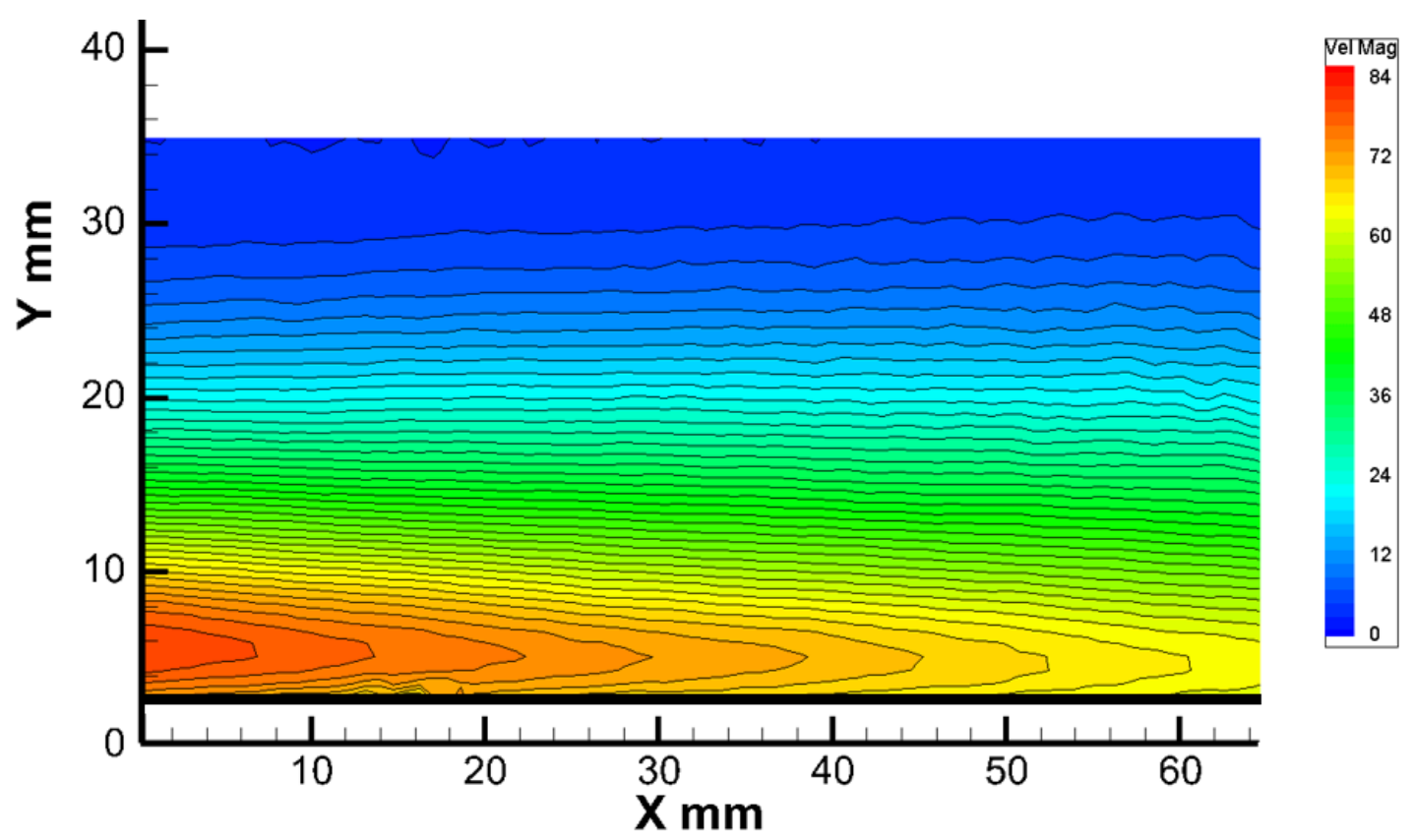

Fig. 17 Close up view of streamwise velocity distribution along $X$ axis over flat plate without PPS Aerostrip

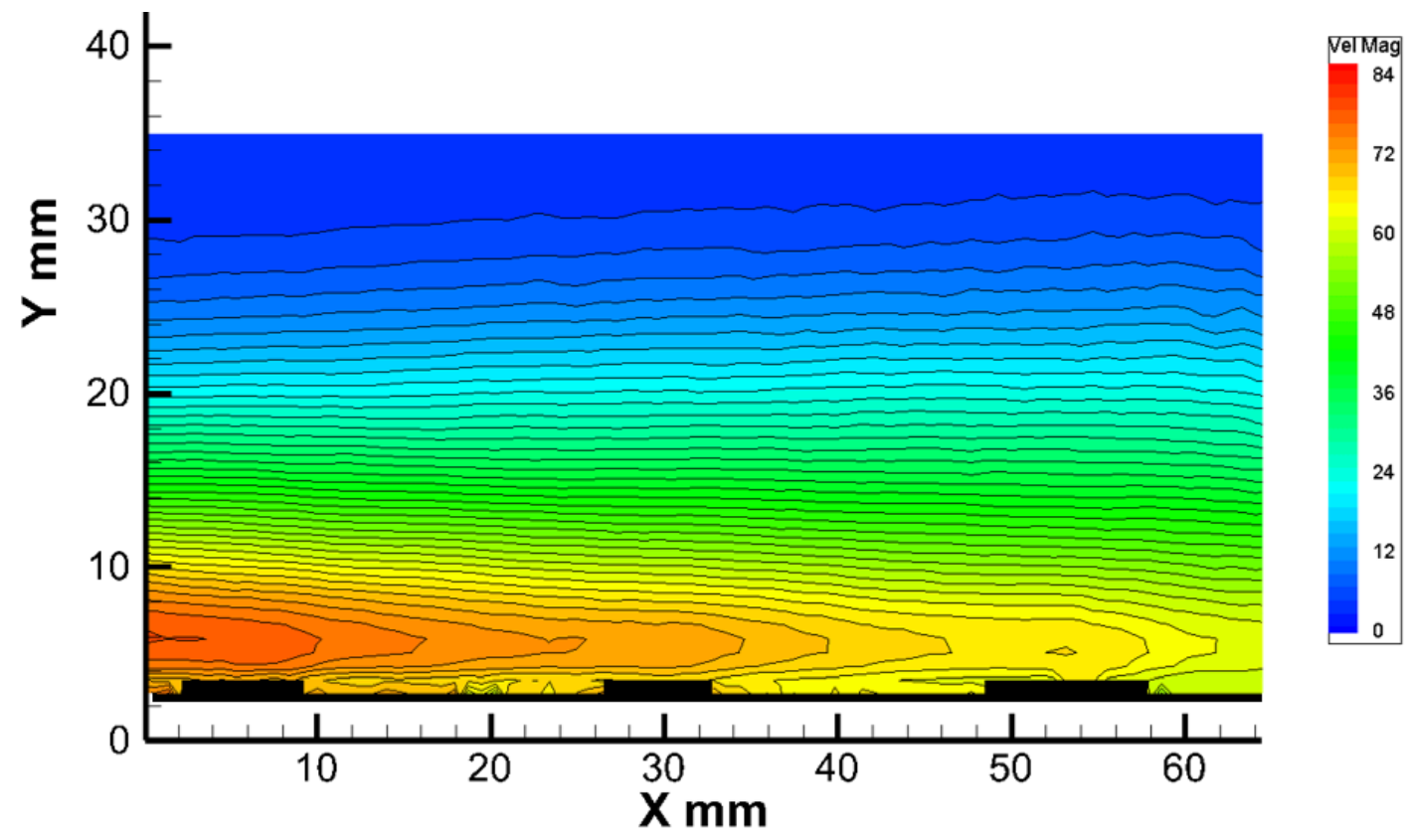

Fig. 18 Close up view of streamwise velocity distribution along $X$ axis over flat plate with PPS Aerostrip

Fig. 19 compares the velocity magnitude at three different heights from the flat plate surface $-2 \mathrm{~mm}, 3 \mathrm{~mm}$ and $5 \mathrm{~mm}$. The velocity magnitude momentarily increases to a higher value as it passes on each sensor and return to 
nominal flat plate value. This is important, because the presence of sensor humps didn't separate the flow, instead it produce a local velocity variation, gradually increasing upstream of the hump and reaches it max velocity at the top of the hump where the sensor is located and in turn decreases the local pressure at the sensor tip. The variation in local velocity diminishes as we move above $5 \mathrm{~mm}$ from the surface.
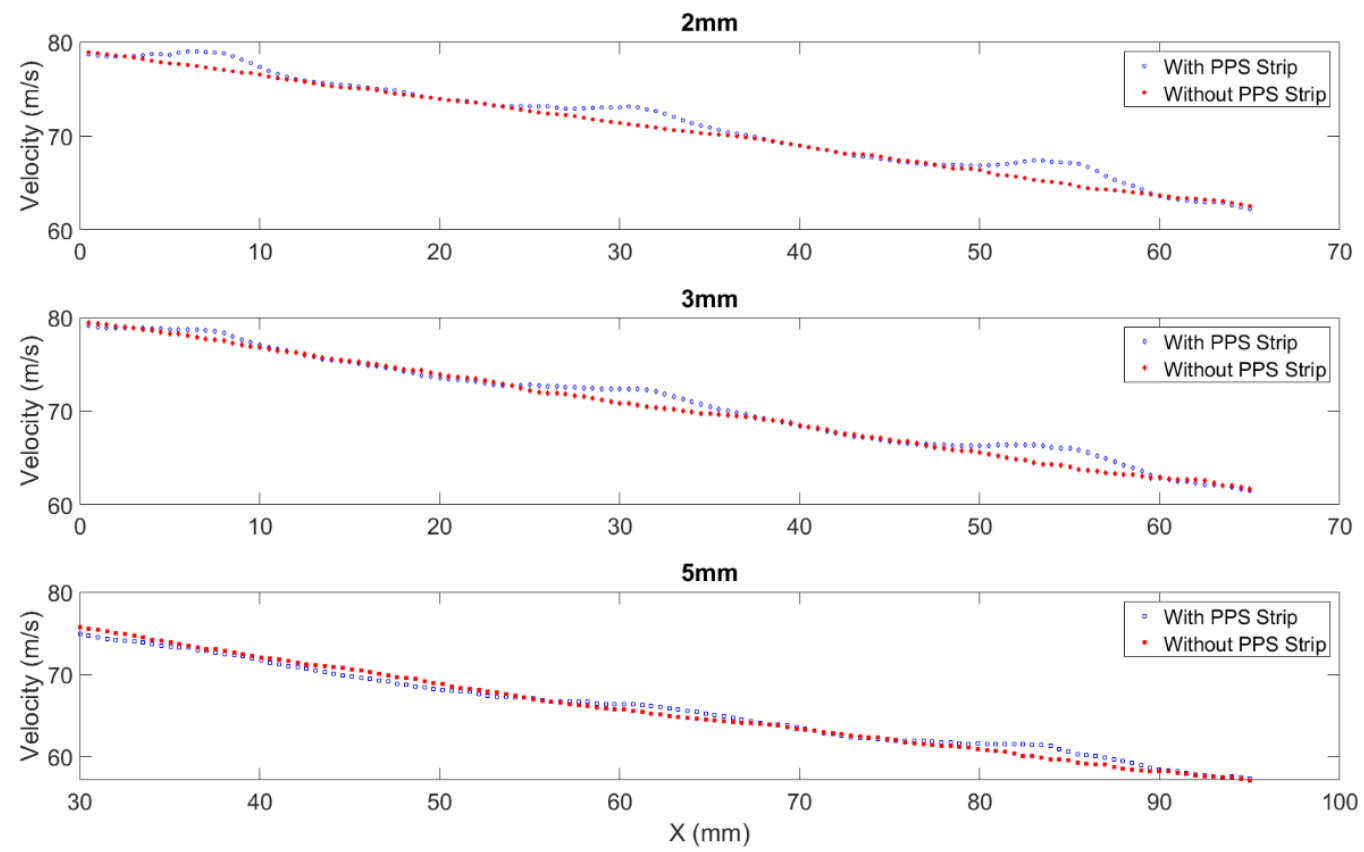

Fig. 19 Comparison of streamwise velocity magnitude at vertical distance of $2 \mathrm{~mm}, 3 \mathrm{~mm}$ and $5 \mathrm{~mm}$ from flat plate surface with and without PPS Aerostrip

\section{Conclusion}

The PPS Aerostrip sensor in this current form captures the pressure distribution over incompressible flow well when compared with the conventional pressure measurement. The results match in static environment, internal flow and external flow, indicating that this is a useful system for most aerodynamic applications. However, the advancement of modern aerodynamics vehicle design poses challenges to develop a sensor system which can be compatible with complex shapes. As shown in this work the sensor correlation is reduced due to misalignment of the straight PPS Aerostrip on the pressure hole curved path of S-Duct side wall and flow measurement at steep convex surfaces, but this remains a useful output as it can lead to more appropriate designs in future. It has been proposed to reduce the sensor element pitch to $8 \mathrm{~mm}$ so it can be used on flow over aerofoil leading edges or in similar kinds of high-pressure gradient environments, where increased spatial resolution is required. Finally, future work shall continue the validation of this type of sensor, likely through a new design iteration with the manufacturer, for use in realistic operating conditions.

\section{Acknowledgment}

This work was supported in part by the CENSIS (Glasgow, UK) in collaboration with PPS UK Limited (Glasgow, UK)

\section{References}


[1] I. Oxyzoglou and I. Nerantzis. "Weight Optimization of a F1 Composite Front Wing" 7th Before Reality Conference. (2017) Thessaloniki.

[2] Zheng Min, Vu Khac Kien \& Liew J.Y. Richard "Aircraft morphing wing concepts with radical geometry change", The IES Journal Part A: Civil \& Structural Engineering, (2010). 3:3, 188-195, DOI: 10.1080/19373261003607972

[3] Jakub Broniszewski \& Janusz Piechna "A fully coupled analysis of unsteady aerodynamics impact on vehicle dynamics during braking”, Engineering Applications of Computational Fluid Mechanics, (2019), 13:1, 623-641, DOI: 10.1080/19942060.2019.1616326

[4] Pressure Profile Systems (PPS Inc., US-CA), https://www.pressureprofile.com, Accessed 28/05/2021.

[5] http://scanivalve.com/products/pressure-measurement/miniature-analog-pressure-scanners/zoc23bminiature-pressurescanner/

[6] https://sensorpros.com/products/druck-dpi-610-615-is-pressure-calibrator

[7] https://www.omega.co.uk/pptst/PXM309-10V.html

[8] Norberg, Christoffer. "An experimental investigation of the flow around a circular cylinder: influence of aspect ratio." Journal of Fluid Mechanics 258 (1994): 287-316

[9] Lee, Jihyeong, Seawook Lee, and Jinsoo Cho. "Effect of Inlet Boundary Layer Suction on Flow Distortion in Subsonic Diffusing S-Duct." International Journal of Aeronautical and Space Sciences 20, no. 4 (2019): 850-857

[10] Wojewodka, Michael M., Craig White, Shahrokh Shahpar, and Konstantinos Kontis. "A review of flow control techniques and optimisation in s-shaped ducts." International Journal of Heat and Fluid Flow 74 (2018): 223-235.

[11] Aslan, Samet, and D. Funda Kurtulus. "Numerical Investigation of an S-Duct Diffuser at Different Inlet Boundary Conditions." In Advances in Sustainable Aviation, pp. 111-128. Springer, Cham, 2018.

[12] Suzuki, Takao. "Effects of a synthetic jet acting on a separated flow over a hump." Journal of Fluid Mechanics 547 (2006): 331-359.

[13] Masad, Jamal A., and Venkit Iyer. "Transition prediction and control in subsonic flow over a hump." Physics of Fluids 6, no. 1 (1994): 313-327. 